\title{
Spinal epidural lipomatosis: presentation of two idiopathic cases and review of the literature
}

\author{
Emmanouel Chatzidakis, George Barlas, Panagiotis Zogopoulos* and Moschos Fratzoglou \\ Department of Neurosurgery, General Hospital of Nikaia - Piraeus “Agios Panteleimon”, Athens, Greece
}

\begin{abstract}
Spinal epidural lipomatosis (SEL) is a rare cause of low back pain and radiculopathy and is usually associated with exogenous steroid therapy and endocrinopathy. The idiopathic form is even more rare and presented mainly in obese patients. SEL appears in the lumbar and thoracic spine and has never been reported in the cervical spine. The deposition of adipose tissue in the spinal canal produces slowly progressing symptoms (low back pain and intermittent claudication) due to compression of the spinal cord and roots. Surgical therapy provides immediate relief, but has a reported mortality rate of over $20 \%$, due to the co-morbidity of these patients. We present two cases of lumbar radiculopathy due to idiopathic spinal epidural lipomatosis. One patient was treated surgically (removal of excessive epidural adipose tissue combined with decompressive laminectomy and foraminotomy), while the other was placed under a low-calorie diet and both improved neurologically.
\end{abstract}

\section{Introduction}

Spinal epidural lipomatosis (SEL) is a pathologic overgrowth of normal epidural fat. It was first described by Lee et al. [1] in 1975 in a 15-year-old patient receiving steroids after renal transplantation. Badami and Hinck [2] in 1982 described the same syndrome in an obese woman who did not receive steroids. SEL is an uncommon disorder, usually associated with high blood steroid level, where accumulation of excessive unencapsulated epidural adipose tissue causes compression of the neural elements within the spinal canal. The main effect exerted by the excessive epidural fat can present as progressive compressive myelopathy or a cauda equina syndrome. It affects the thoracic (58$61 \%)$ or lumbar (39-42\%) spine and has never been reported in the cervical spine, possibly due to the small amount of epidural fat in this part of the spine [3].

Hypertrophy of the adipose tissue in the spinal epidural space has been associated with the exogenous administration of steroids in approximately $75 \%$ of cases $[3,4]$. In these cases the disorder can be attributed to the abnormal adipose deposition observed in patients with high steroid blood level. It has also been observed in association with Cushing disease, Cushing syndrome [4], hypothyroidism [5], pituitary prolactinoma [6], radiotherapy and obesity [7]. In a small number of cases no obvious cause has been detected and the disorder is considered idiopathic $[4,5,8]$. Most of these patients were morbidly obese. Idiopathic epidural lipomatosis occurs with equal frequency in the thoracic and lumbar spine [7]. SEL appears more commonly in men than women (3/1 ratio) [9]. The mean age of patients with SEL is 43 years, but tents to appear at a younger age in males [9]. We present two interesting cases of idiopathic SEL and their therapeutic approach.

\section{Materials and methods}

\section{Case 1}

A 70-year-old male presented with progressively worsening low back pain and intermittent claudication of the lower limbs over the last year. He complained of inability to walk more than 20 meters and then having to rest for his leg pain to subside. He had received medical treatment with non-steroid anti-inflammatory medication for more than six months with moderate success.

Physical examination revealed obesity $(170 \mathrm{~cm}, 130 \mathrm{~kg}, \mathrm{BMI}$ $44.98 \mathrm{~kg} / \mathrm{m}^{2}$ ) without local tenderness in the lower back. He was able to move without distress in the examination room and trunk and lower limb sensation was normal. Muscle strength was normal and the straight leg raising test was negative bilaterally. Both patellar and Achilles reflexes were almost absent bilaterally. He refused bowel or bladder dysfunction, but admitted loss of erection. In the medical history he reported mild arterial hypertension under treatment and diabetes mellitus treated with diet and medication. He was suffering of chronic obstructive pulmonary disease, and received treatment with inhalation bronhodilators. No history of exogenous steroid administration was revealed. He had received various combinations of analgesics and anti-inflammatory medication for his lower back pain. Lumbar X-ray examination revealed only mild reduction of lordosis in the lateral view. A CT-scan was performed revealing an extradural low density mass compressing the dural sac. The lumbar MRI scan showed increased deposition of extradural fat circumferentially compressing and causing polygonal deformation of the dural sac (Figure 1). The diagnosis of lumbar epidural lipomatosis was made and the patient was placed under a low calorie diet with moderate success. Surgical treatment could not be applied because the patient was considered of high risk for general anesthesia.

Correspondence to: Panagiotis Zogopoulos, MD, Department of Neurosurgery, General Hospital of Nikaia - Piraeus "Agios Panteleimon", 69 Vosporou Str., Athens, Greece, PO Box: 10444, Tel: (+30)210-5128632; Mob: $(+30) 6976-$ 053555; Fax: (+30)210-5128632; E-mail: p.zogopoulos@yahoo.com

Key words: idiopathic, obesity, radiculopathy, spinal epidural lipomatosis

Received: March 08, 2015; Accepted: April 09, 2015; Published: April 12, 2015 


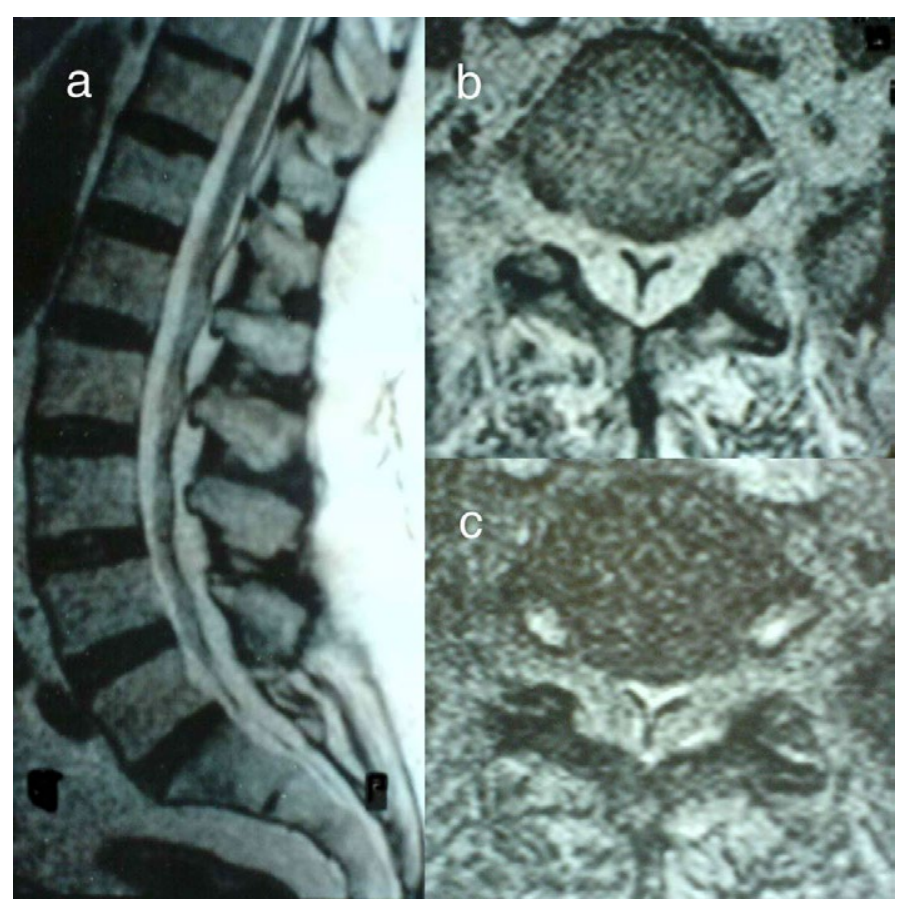

Figure 1. MR imaging appearance of epidural lipomatosis in case 1. a: Midline Sagittal T1-weighted MR image of the lumbar spine. Narrowing of the thecal sac is prominent, most severe below the L4-L5 disc space, unrelated to osseous or soft tissue canal lesions. b: Axial T1 and c: Axial T2-weighted MR images showing the Y-shaped dural sac deformation whereas the spinal canal has normal antero-posterior diameter.

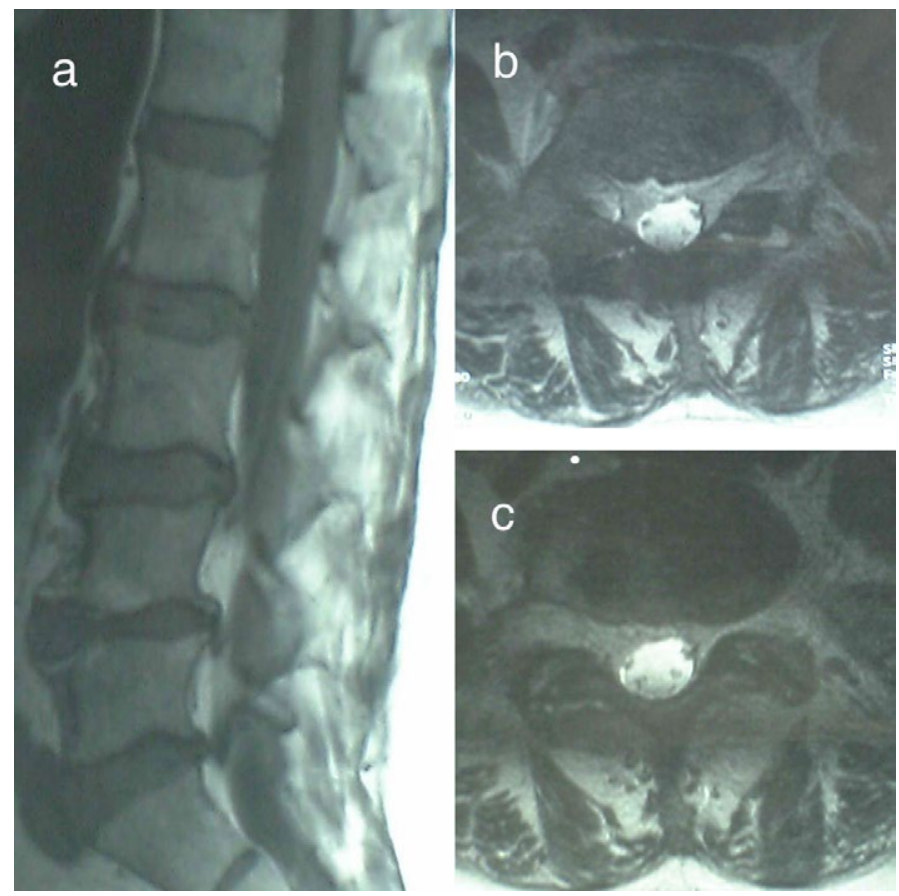

Figure 2. MR imaging appearance of epidural lipomatosis in case 2. a: Midline Sagittal T1-weighted MR image of the lumbar spine showing high-signal intensity fat surrounding the dural sac below the L3-L4 level in the spinal canal. b: Axial T2-weighted MR image showing fat surrounding the dural sac below the L3-L4 level in the spinal canal. c: Axia T2-weighted MR image at the L3-L4 level showing fat infiltration of the lateral epidural space going across the intervertebral foramen.

\section{Case 2}

A 70-year-old male presented with low back pain, foot drop on the left and multiradicular symptoms with hypesthesia and pain of both lower limbs. He had no symptoms of the upper limbs. The general medical history was negative. He suffered of no chronic illness, nor did he receive any medication. Neuroimaging studies revealed extensive degeneration with multiple intervertebral disc herniations and foraminal stenosis, as well as the presence of excessive epidural adipose tissue (Figure 2). Neurophysiological studies confirmed the multiradicular involvement.

The excessive adipose tissue was removed surgically and a L4-L5 discectomy and multiple foraminotomies were performed. Histological examination of the surgical specimen revealed normal adipose tissue. The patient noted immediate postoperative improvement of the radicular symptoms, but no improvement of left foot dorsiflection. A few months later the patient presented with worsening of his clinical symptomatology and the diagnosis of Charcot disease was made. $\mathrm{He}$ died within a few months.

\section{Discussion}

SEL is a rare cause of back pain often long before neurological symptoms appear. Slowly progressive weakness of the lower limbs, numbness, paresthesias or radicular symptoms can be found. Bladder and bowel incontinence appear rarely. Deep tendon reflexes are commonly brisk and pinprick sensation may be decreased $[4,9]$. The symptomatology is suggestive of the level of the lesion with thoracic levels producing myelopathic symptoms and lumbar levels radicular.

The symptoms of idiopathic SEL are typically of gradual onset and similar to the steroid-induced [4]. Spinal cord compression is most commonly due to impingement by disk or bone. They usually produce focal symptoms. In SEL the narrowing extends along several vertebrae and symptoms are not confined to one level. Hemorrhage, neoplasm and infection can have similar symptomatology. The elevated erythrocyte sedimentation rate, that can be seen, is nonspecific and can occur in patients with SEL receiving high dose steroids, as treatment for autoimmune or chronic inflammatory diseases. In such cases the low attenuation of fat in the CT-scan can differentiate SEI from these conditions. Less common causes of cord compression like synovial cysts, extramedullary hematopoiesis and arachnoid cysts can be differentiated from SEL on the basis of their imaging characteristics.

Epidural lipomatosis as a cause of myelopathy should be considered when:

- A complete posterior block is seen at myelography,

- CT or MR imaging reveals only fat contiguous to a ventrally displaced dural sac,

- There is a history of chronic steroid use,

- There are myelopathic or radicular symptoms referable to the level of the abnormality,

- There are no other structural lesions that could explain the symptoms and imaging findings.

MRI is the study of choice for the diagnosis of SEL [4,10]. Epidural adipose tissue of greater than $7 \mathrm{~mm}$ thickness is considered a diagnostic criterion for SEL [7]. Adipose tissue gives a high intensity signal on T1WI and intermediate signal on T2WI. Canal obstruction and obliteration of CSF spaces can be appreciated with this study. The shape of the dural sac shows changes in the successive transverse sections of the lumbar spine. It is more often polygonal (hexagonal or pentagonal) in the upper lumbar spine, turning to an inverted triangular or Y shape 
in the lower spine that is considered pathognomic in lumbar axial imaging (the "Y" -sign). CT-scan, that usually precedes the MRI, can be used to differentiate the cause of compression since the density of adipose tissue on CT-scans, ranges between 80 to $120 \mathrm{HU}$ [9]. Plain $\mathrm{X}$-ray films cannot diagnose SEL, but can only be helpful to exclude other causes of back pain, like degenerative disease and tumors.

The therapeutic approach of patients with SEL is individualized and can be either surgical or conservative. Concerning the conservative therapy, weaning of patients from steroids and weight loss can be successful [4]. In many cases though, patients receiving steroids for chronic illnesses may not tolerate weaning. Weight loss can provide treatment in obese patients with no other cause for SEL. Patients not receiving steroids should undergo thorough endocrinological evaluation to exclude possible endogenous corticosteroid overproduction.

Concerning the surgical treatment, decompressive laminectomy with resection of epidural adipose tissue is the most successful surgical intervention $[9,10]$. The majority of these patients report immediate improvement of their symptoms. Patients with symptoms due to cauda equina compression make a better recovery after surgical decompression [9]; patients receiving lower doses of steroids preoperatively make better neurological recovery [9] while patients with idiopathic SEL tend to have better prognosis [8]. Fessler et al. [3] have reported $22 \%$ mortality rate in these patients within a year after surgical decompression. Although the procedure carries little risk, these patients' co-morbidity and medical problems complicate the postoperative management and morbidity. They recommend conservative treatment, reserving surgical decompression for patients in whom other measures fail.

\section{References}

1. Lee M, Lekias J, Gubbay SS, Hurst PE (1975) Spinal cord compression by extradural fat after renal transplantation. Med J Aust 1: 201-203. [Crossref]

2. Badami JP, Hinck VC (1982) Symptomatic deposition of epidural fat in a morbidly obese woman. AJNR Am J Neuroradiol 3: 664-665. [Crossref]

3. Fessler RG, Johnson DL, Brown FD, Erickson RK, Reid SA, et al. (1992) Epidural lipomatosis in steroid-treated patients. Spine (Phila Pa 1976) 17: 183-188. [Crossref]

4. Dumont-Fischer D, Rat AC, Saidenberg-Kermanac'h N, Laurent S, Cohen R, et al (2002) Spinal epidural lipomatosis revealing endogenous Cushing's syndrome. Joint Bone Spine 69: 222-225. [Crossref]

5. Stambough JL, Cheeks ML, Keiper GL (1989) Nonglucocorticoid-induced lumbar epidural lipomatosis: a case report and review of literature. J Spinal Disord 2: 201-207. [Crossref]

6. Fujisawa H, Hasegawa M, Tachibana O, Yamashita J (2002) Spinal epidural lipomatosis associated with pituitary macroprolactinoma. Acta Neurochir (Wien) 144: 213-214. [Crossref]

7. Kumar K, Nath RK, Nair CP, Tchang SP (1996) Symptomatic epidural lipomatosis secondary to obesity. Case report. J Neurosurg 85: 348-350. [Crossref]

8. Payer M, Van Schaeybroeck P, Reverdin A, May D (2003) Idiopathic symptomatic epidural lipomatosis of the lumbar spine. Acta Neurochir (Wien) 145: 315-320. [Crossref]

9. Roy-Camille R, Mazel C, Husson JL, Saillant G (1991) Symptomatic spinal epidural lipomatosis induced by a long-term steroid treatment. Review of the literature and report of two additional cases. Spine (Phila Pa 1976) 16: 1365-1371. [Crossref]

10. Fan CY, Wang ST, Liu CL, Chang MC, Chen TH (2004) Idiopathic spinal epidural lipomatosis. J Chin Med Assoc 67: 258-261. [Crossref]

Copyright: (C2015 Chatzidakis E. This is an open-access article distributed under the terms of the Creative Commons Attribution License, which permits unrestricted use, distribution, and reproduction in any medium, provided the original author and source are credited. 